\title{
Multimode EDFA performance in mode-division multiplexed transmission systems
}

\author{
Y. Jung, ${ }^{1 *}$ V. A. J. M. Sleiffer, ${ }^{2}$ B. Inan, ${ }^{3}$ M. Kuschnerov, ${ }^{4}$ V. Veljanovski, ${ }^{4}$ B. Corbett, ${ }^{5}$ R. Winfield, ${ }^{5}$ Q. Kang, \\ ${ }^{1}$ A. Dhar, ${ }^{1}$ J. Sahu, ${ }^{1}$ F. Poletti, ${ }^{1}$ S. -U. Alam, ${ }^{1}$ D. J. Richardson ${ }^{1}$ \\ ${ }^{1}$ Optoelectronics Research Centre, University of Southampton, Southampton, SO17 1BJ, UK \\ ${ }^{2}$ COBRA institute, Eindhoven University of Technology, The Netherlands \\ ${ }^{3}$ Institute of Communications Engineering, Technische Universität München, Arcisstrasse 21 D-80333, Munich, Germany. \\ ${ }^{4}$ Nokia Siemens Networks GmbH \& Co. KG, Munich, Germany \\ ${ }^{5}$ Tyndall National Institute, Cork, Ireland \\ ymj@orc.soton.ac.uk
}

\begin{abstract}
We report a detailed study on the system performance of a two-mode group EDFA. In particular we quantify how the gain spectrum and BER performance are affected by input signal and pump power as required in the execution of our ongoing MDM transmission experiments. OCIS codes: (060.0060) Fiber optics and optical communications; (060.2320) Fiber optics amplifiers and oscillators.
\end{abstract}

\section{Introduction}

To date single mode fibers (SMFs) have been used as the transmission medium of choice in long-haul telecommunication systems, with the use of multimode fibers (MMFs) restricted to short distance (e.g. local area) networks due to their severe modal dispersion which grossly limits the transmission bandwidth. However, driven by fears of a future "capacity crunch", major interest is developing in the potential use of "mode division multiplexing (MDM)" in which selective mode excitation and detection schemes are used to define distinguishable information channels within a few mode fiber (FMF) [1-3]. Since the modes are orthogonal, it is in principle possible to increase the capacity in accordance with the number of modes supported by the FMF. Digital signal processing can be utilized to mitigate transmission impairments caused by mode-coupling in the MDM systems and thereby to substantially increase the achievable transmission distance. However, in order to be useful in long-haul systems a practical, high performance FMF amplifier is essential. In our recent work [4], we successfully demonstrated a multimode (two-mode group) erbium-doped fiber amplifier (MM-EDFA) for MDM applications providing simultaneous modal gains of $\sim 20 \mathrm{~dB}$ for different pair-wise combinations of spatial and polarization modes. However, in order to reliably use this device in full data transmission experiments a far more detailed understanding of how the amplifier performs under more fully loaded conditions (both in terms of DWDM channels across the Cband and the full set of supported modes) is required. In this paper, we report a detailed characterization of how the input signal power and pump power levels affect the spectral gain flatness and bit error rate (BER) performance and the optimum condition required to obtain the minimum power penalty are discussed. Note that the following characterization of the MM-EDFA was successfully used in the 96-channel, $73.7 \mathrm{Tbit} / \mathrm{s}$ MDM transmission experiments reported in our recent ECOC 2012 postdeadline paper [2].

\section{Experimental setup}

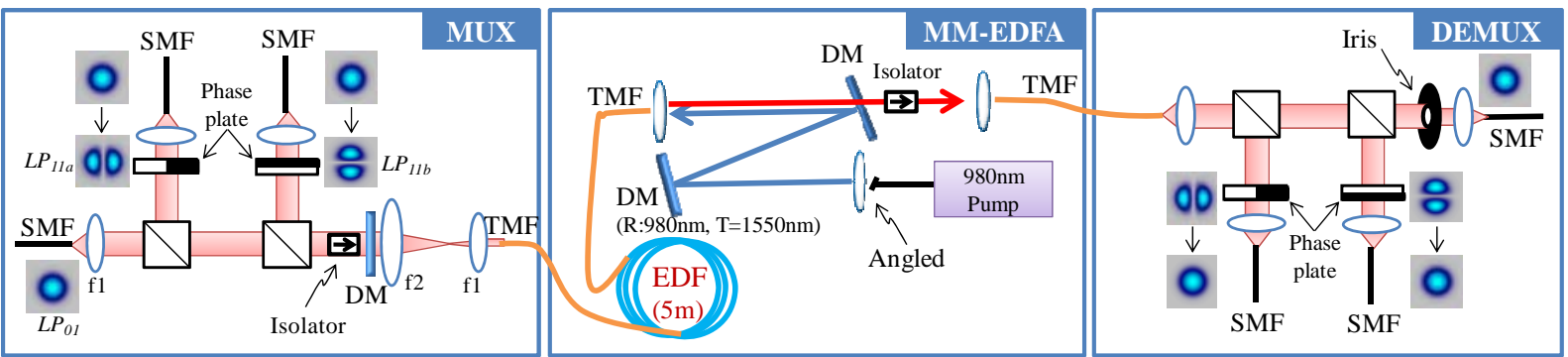

Fig. 1. Schematic design of mode multiplexer (MUX), multimode erbium doped fiber amplifier (MM-EDFA) and mode demultiplexer (DEMUX).

To evaluate the MM-EDFA performance in a transmission system, 17 external cavity lasers at distinct wavelength across the C-band were multiplexed and modulated with 256-Gb/s DP-16QAM using an IQ-modulator and polarization-multiplexing stage. The data stream was split up into three equally powered signals and fed to the mode multiplexer (MUX) as described in [2]. As shown in Fig. 1 , three spatial mode channels $\left(L P_{01}, L P_{11 a}, L P_{11 b}\right)$ were 
multiplexed using free space optics and phase plates. Polymethyl methacrylate (PMMA) phase plates were used to selectively excite the $L P_{11}$ modes from an incident $L P_{01}$ mode and the light was combined using two beam splitters and coupled into a short 10m-length of passive two mode fiber (TMF). To provide clean mode-excitation, a telecentric lens system ( $\mathrm{f} 1=3.1 \mathrm{~mm}, \mathrm{f} 2=125 \mathrm{~mm}$ ) was adopted and an extinction ratio of $>25 \mathrm{~dB}$ was achieved. A dichroic mirror (980nm reflection and 1550nm transmission) was used to reject any unabsorbed pump power while a polarization-insensitive isolator was used to suppress any signal light propagation in the backward direction. The passive TMF was then spliced directly to a 5m-length of mode-matched $\mathrm{Er}^{3+}$-doped active fiber [4-6] and a 980nm pump laser was free-space coupled into the TM-EDF using two dichroic mirrors. The amplified output was coupled into another 10m-length of TMF and was demultiplexed into three spatial channels using mode DEMUX.

\section{Gain spectra and BER performance of the MM-EDFA}

(a)

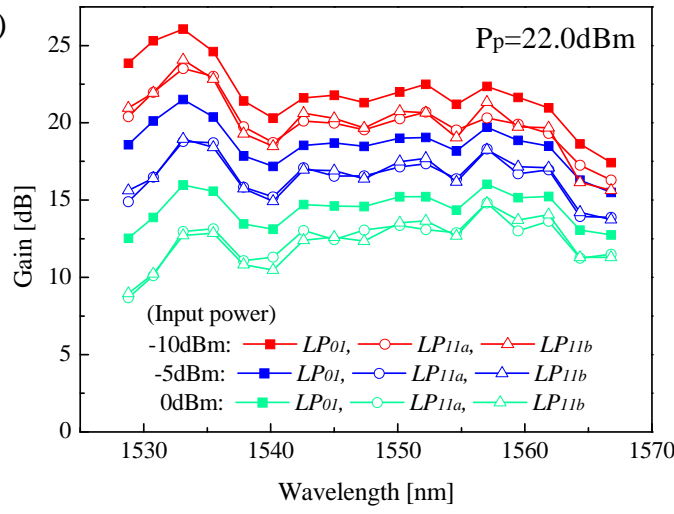

(b)

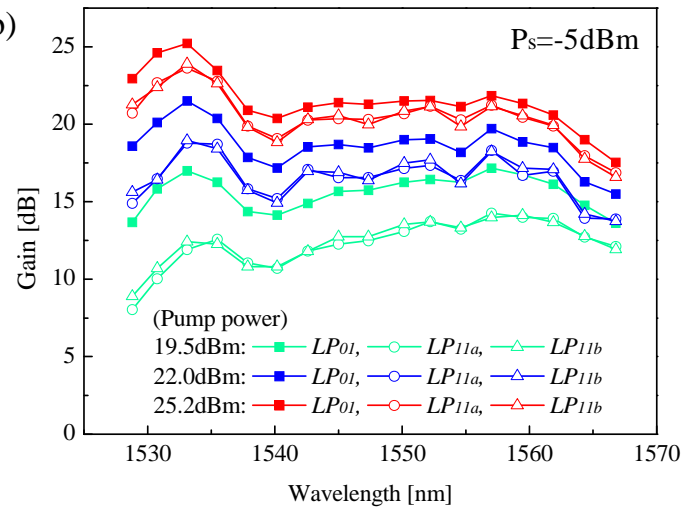

Fig. 2. Measured gain spectrum characteristics for (a) different input signal powers $\left(P_{s}\right)$ and (b) pump powers $(P p)$.

First, the gain spectrum of the MM-EDFA was investigated for different input signal powers per spatial mode (including both polarizations and 17 channels) as shown in Fig. 2. An offset pump launch condition was applied to maximize the gain for the $L P 11$ modes and the total launched pump power was fixed at $22 \mathrm{dBm}$. For an input signal power of $-10 \mathrm{dBm}$ per mode, the average gain across the full C-band was $22 \mathrm{~dB}$ for the $L P_{01}$ and $20 \mathrm{~dB}$ for the $L P_{11}$ modes. Both modes experience gain reduction with an increase in input signal powers however the gain flatness improves as the EDFA becomes more deeply saturated. Fig. 2(b) shows the gain spectra of the amplifier for different pump powers at a fixed input signal power of $-5 \mathrm{dBm}$ per mode. As the pump power increases from $19.5 \mathrm{dBm}$ to $25.2 \mathrm{dBm}$, both the modal gains were increased and the differential modal gain was improved from $5 \mathrm{~dB}$ to $1 \mathrm{~dB}$. Note also that the gain peak shifts from $1560 \mathrm{~nm}$ to $1530 \mathrm{~nm}$.

(a)

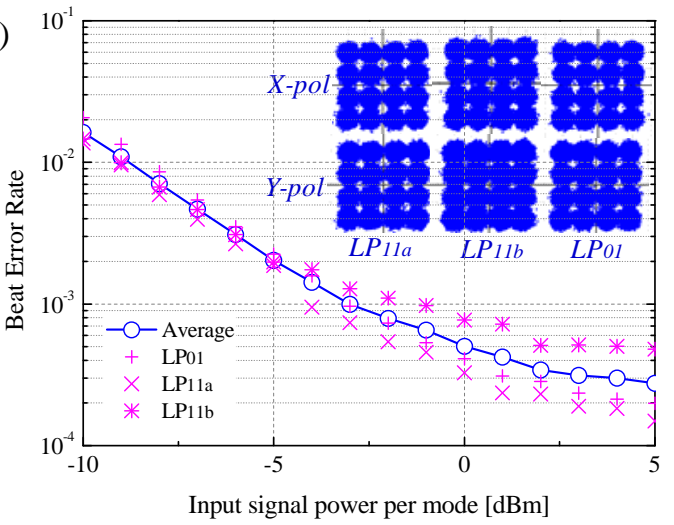

(b)

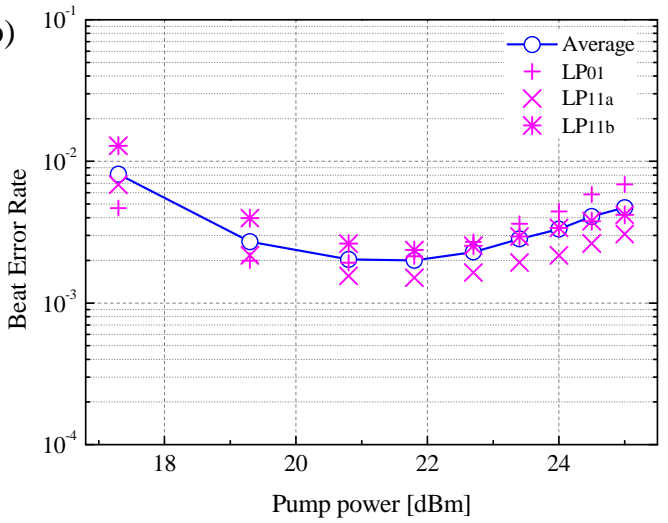

Fig. 3. BER performance of the MM-EDFA as a function of (a) input signal power per mode and (b) launched pump power. The constellations are the recovered 256-Gb/s DP-16QAM at an input signal of 5dBm per mode.

To evaluate the bit error rate (BER) performance, the three output ports of the DEMUX were plugged into 50GHz tunable optical filters and commercial coherent receivers. The samples obtained from the digital sampling scopes were recorded and processed offline using data-aided digital signal processing. Fig. 3(a) shows the BER 
measurement results of the MM-EDFA as a function of the input signal power per mode at a fixed pump power of $22 \mathrm{dBm}$. It is clearly seen that the BER performance is highly dependent on the input signal power and that too low input signal power leads to a rapid degradation in the BER, due to the increased level of ASE (reduced optical signal to noise ratio (OSNR)). We also tested the BER performance of the amplifier according to various pump powers at a fixed input signal power of $-5 \mathrm{dBm}$ per mode. As depicted in Fig. 3(b), the experimental BER curve shows that an optimum pump power exists giving the best BER performance for a given input signal power. Generally, the higher the population inversion, the lower the noise figure of the amplifier. Thus, at low pump powers $(<22 \mathrm{dBm})$, the BER was improved by increasing the pump power due to the increased population inversion, however at higher pump powers $(>22 \mathrm{dBm})$ the BER begins to degrade due to the increased ASE from the MM-EDFA.

(a)

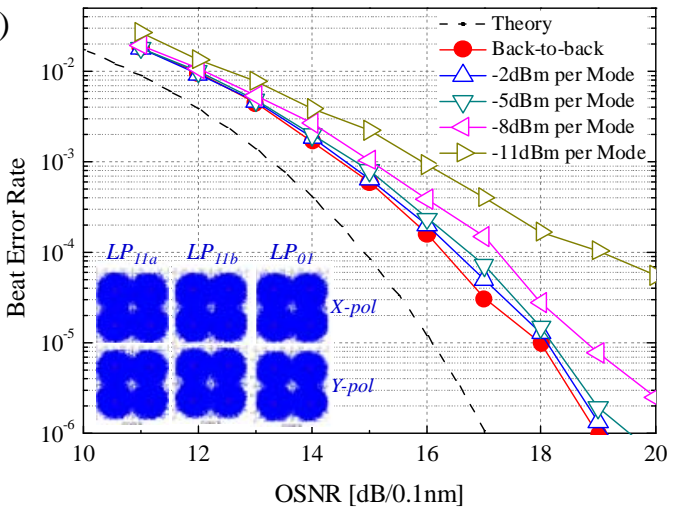

(b)

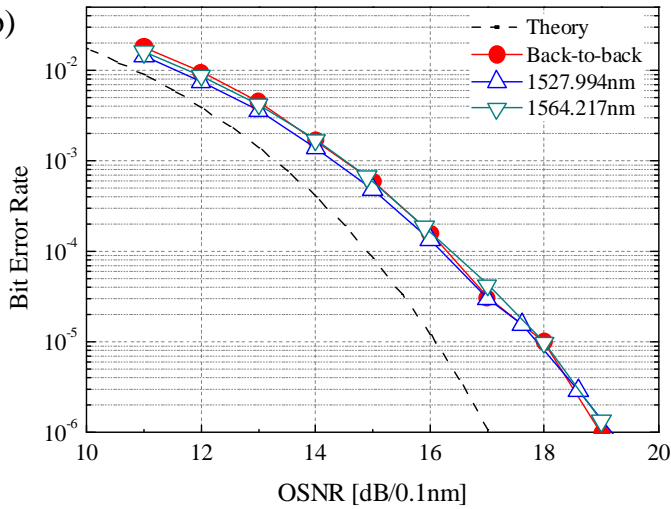

Fig. 4. (a) The measured BER performances at $1550.2 \mathrm{~nm}$ for various input signal power into the MM-EDFA and (b) measured BER at the edge channels of C-band (1527.994nm and $1564.217 \mathrm{~nm})$ at the input signal power of $-5 \mathrm{dBm}$. The constellations are the recovered 112Gb/s DP-QPSK for an input signal power of $-5 \mathrm{dBm}$ per mode with an OSNR of $15 \mathrm{~dB} / 0.1 \mathrm{~nm}$.

Fig. 4(a) shows the averaged BER curves of all spatial modes $\left(L P_{01}, L P_{11 a}\right.$ and $\left.L P_{11 b}\right)$ for a $112 \mathrm{~Gb} / \mathrm{s}$ DP-QPSK signal at the center wavelength of the C-band $(1550.2 \mathrm{~nm})$. As described in Fig. 3(a), the overall BER performance is strongly dependent on the input signal power into the MM-EDFA which affects the output OSNR. The induced power penalty from the MM-EDFA was $0.1,0.2,0.5$ and $1.5 \mathrm{~dB}$, respectively, for the $-2,-5,-8$ and $-11 \mathrm{dBm}$ input signal power per mode cases compared to the back-to-back condition. The observed power penalty is small but it could be further reduced by applying a gain flattening filter after the amplifier and reducing remnant Fresnel reflections from the fiber end facets. We further tested the BER performance of our MM-amplifier at the edge channels of the C-band (1527.994nm and 1564.217nm). As shown in Fig. 4(b), the edge channel also shows a good power penalty less than $0.2 \mathrm{~dB}$ at the input signal power of $-5 \mathrm{dBm}$ per mode.

\section{Conclusions}

We have experimentally investigated the system performance of a multimode erbium-doped fiber amplifier in a mode-division multiplexing system. The overall BER performance is highly dependent on the input signal power into the MM-EDFA and low input signal powers lead to a degradation of the BER due to ASE build-up. For a given input signal power there exists an optimum pump power that provides the best BER performance. Such detailed studies of MM-EDFA performance have enabled very high capacity DWDM MDM transmission experiments and should also allow for further amplifier optimization in due course.

\section{Acknowledgement}

This work was supported by the European Communities $7^{\text {th }}$ Framework Programme under grant agreement 258033 (MODE-GAP).

\section{References}

[1] R. Ryf et al., , J. of Lightwave Technol. 30, 521-531 (2012).

[2] V.Sleiffer et al., in Proceedings of ECOC'12, Th.3.C.4 (2012).

[3] N. Bai et al., Optics Express 20, 2668-2680 (2012).

[4] Y. Jung et al Optics Express 19, B952-B957 (2011).

[5] Q. Kang et al., "Optics Express 20, 20835-20843 (2012).

[6] Y. Jung et al., in Proceedings of ECOC'12, Tu.3.F.5 (2012). 